\title{
Reconstructing Dark Energy Potentials From Parameterized Deceleration Parameters*
}

\author{
Wang Yu-Ting, Xu Li-Xin ${ }^{\dagger}$, Lü Jian-Bo, and Gui Yuan-Xing \\ School of Physics and Optoelectronic Technology, \\ Dalian University of Technology, Dalian, Liaoning 116024, P. R. China
}

\begin{abstract}
In this paper, the properties of dark energy are investigated according to the parameterized deceleration parameter $q(z)$, which is used to describe the extent of the accelerating expansion of the universe. The potential of dark energy $V(\phi)$ and the cosmological parameters, such as the dimensionless energy density $\Omega_{\phi}, \Omega_{m}$, and the state parameter $w_{\phi}$, are connected to it. Concretely, by giving two kinds of parameterized deceleration parameters $q(z)=a+\frac{b z}{1+z}$ and $q(z)=\frac{1}{2}+\frac{a z+b}{(1+z)^{2}}$, the evolution of these parameters and the reconstructed potentials $V(\phi)$ are plotted and analyzed. It's found that the potentials run away with the evolution of universe.
\end{abstract}

Keywords: dark energy(DE), deceleration parameter, reconstructed potential PACC: 9880

\section{Introduction}

Since 1998, the type Ia supernova (SNe Ia) observations [1] show that the expansion of our universe is speeding up rather than slowing down. During these years from that time, many additional observational results, including current Cosmic Microwave Background (CMB) anisotropy measurement from WMAP [2], and the data of the Large Scale Structure (LSS) [3], also strongly support this suggestion. These observational results have received considerable recognition. In order to understand the mechanism of the accelerating expansion of the universe, the authors have done plenty of work, which can be divided into two categories: on the one hand, we can carry on the revision to the geometry items at the left-handed side of the Einstein field equation $\left(R_{\mu \nu}-\frac{1}{2} g_{\mu \nu} R=4 \pi G T_{\mu \nu}\right)$, and the famous work is $f(R)$ theory [4]; On the other hand, we can modify the material item at the righthanded side of this equation. Therefore, many workers have always been making an effort to look for the matter that can lead to this evolutive history of the universe. By combining cosmic observations, it's implied that an exotic energy component, which is called dark energy (DE) and has negative pressure, exists in the universe and its density accounts for two-thirds of the total energy density. In addition, there have been some studies which are accomplished to reconcile the two methods [5]. In the case of the latter, numerous models about DE are proposed in order to clarify the essence of DE. The simplest one is the cosmological constant $\Lambda$, with the equation of state (EOS) $w=\frac{P}{\rho}=-1$. However, it confronts with two difficulties: the fine-tuning problem and the cosmic coincidence problem. An alternative offer is the dynamical DE to overcome the difficulties above. In this class of models, the energy form is provided by an evolving, scalar field $\phi$, with a self-interaction potential $V(\phi)$, such as quintessence [6], phantom [7], quintom [8] and so forth. Furthermore, there are generalized Chaplygin gas (GCG) model [9], Holographic DE [10], high dimension theories [11], etc. Considering more and more DE models, obviously it is very meaningful to explore the properties of $\mathrm{DE}$ in a model independent manner [12]. Usually, we are able to parameterize the state parameter $w(z)$ [13], the energy density $\rho(z)$ [14], or the deceleration parameter $q(z)$ [15] by using cosmological observations.

In view of the dynamical DE models, we can reconstruct the potential $V(\phi)$ from supernova observations [16]. The disadvantage of this kind of methods is that we need to give a concrete form of a potential, which is usually selected to describe the desired properties of DE as well as possible, or an expanding form of a potential. Then, there have been authors reconstructing the potential from the parameterized parameters without assuming its form, such as the state parameter $w(z)$ [17], and the energy density $\rho(z)$ [18], both of which are used to describe the properties of DE. When we give parameterized parameters $w(z)$

\footnotetext{
*Project supported by the National Natural Science Foundation of China (Grant Nos 10573003 and 10703001), and Specialized

Research Fund for the Doctoral Program of Higher Education (Grant No 20070141034).

†E-mail:lxxu@dlut.edu.cn
} 
and $\rho(z)$, to some extent we have limited some properties of DE. On the basis of the previous researches, in turn we can think of reconstructing the potential by a parameter which can directly reflect the transition from the decelerating expansion to the accelerating expansion along with the evolving universe. Based on this consideration, in this paper we will reconstruct the potential of DE according to the parameterized parameter $q(z)$, being positive in the past and negative at present with the history of the universe, which accords with our idea.

Performing a comparison to the previous means, ours has its own advantages. Firstly, according to the expressions of $H(z)=H_{0} \exp \int_{0}^{z}(1+q(u)) d \ln (1+$ $u)$ and $H^{2}(z)=H_{0}^{2}\left[\Omega_{m 0}(1+z)^{3}+\left(1-\Omega_{m 0}\right)(1+\right.$ $\left.z)^{3[1+w(z)]}\right]$, we can see that the constructed Hubble parameter $H(z)$ is independent on cosmological quantity $\Omega_{m 0}$ from the deceleration parameter $q(z)$. And it is well known that Hubble parameter $H(z)$ is an observational quantity, then the cosmological quantities can be affected by $\Omega_{m 0}$ when we constrain them by combining the observational data [19]. But this problem disappears in the case of the deceleration parameter $q(z)$. Secondly, it's known that the state parameter $w(z)$ and the energy density $\rho(z)$ are made use of describing the properties of DE. In other words, giving a parameterized state parameter $w(z)$ or a parameterized energy density $\rho(z)$ designates that we have assumed some properties of DE. For our method, we reconstruct the potential $V(\phi)$ by the parameterized deceleration parameter $q(z)$ without hypothesizing any evolutive mode of DE and probe DE completely grounding on the whole cosmological evolvement.

\section{Construction of the potential and the evolution of cosmolog- \\ ical parameters}

Considering a spatially flat FRW cosmological model, which consists of two components: the nonrelativistic matter and the dynamical DE, we utilize a spatially homogeneous scalar field $\phi$ to describe DE.

With the metric $d s^{2}=-d t^{2}+a^{2}(t)\left[d r^{2}+r^{2}\left(d \theta^{2}+\right.\right.$ $\left.\left.\sin ^{2} \theta d \phi^{2}\right)\right]$, the Einstein field equation can be written as

$$
\frac{\ddot{a}}{a}=-\frac{1}{6 M_{p l}^{2}}\left(\rho_{m}+\rho_{\phi}+3 P_{\phi}\right),
$$

where, $H=\frac{\dot{a}}{a}$ is the Hubble parameter, $\rho_{m}$ is the matter density and $M_{p l} \equiv(8 \pi G)^{-\frac{1}{2}}$ is the reduced Planck mass. The energy density $\rho_{\phi}$ and the pressure $P_{\phi}$ of the evolving scalar field $\phi$ are respectively given by

$$
\begin{aligned}
& \rho_{\phi}=\frac{1}{2} \dot{\phi}^{2}+V(\phi), \\
& P_{\phi}=\frac{1}{2} \dot{\phi}^{2}-V(\phi),
\end{aligned}
$$

where, the dot above $\phi$ designates its derivative with respect to the time $\mathrm{t}$ and $V(\phi)$ is the potential of the evolving scalar field $\phi$. From the equations above, we can easily obtain

$$
\begin{aligned}
& V(\phi)=\frac{1}{2}\left(\rho_{\phi}-P_{\phi}\right), \\
& \dot{\phi}^{2}=\rho_{\phi}+P_{\phi} .
\end{aligned}
$$

Obviously equation (1) can yield $\rho_{\phi}=3 M_{p l}^{2} H^{2}-$ $\rho_{m}$, while the equation (1) and (2) can be combined to result in $P_{\phi}=\left(q-\frac{1}{2}\right) 2 M_{p l}^{2} H^{2}$, where, $q \equiv-\frac{\ddot{a}}{a H^{2}}$ is the deceleration parameter. Using $\rho_{m}=\rho_{m 0}(1+z)^{3}$, where, $\mathrm{z}$ is the redshift, and the relation between the scale factor and the redshift $\mathrm{z}, a(t)=\frac{1}{1+z}$ with $a\left(t_{0}\right)=1$, the equations (5) and (6) can be rewritten as

$$
\begin{aligned}
& V(\phi(z))=\rho_{m 0}\left[\frac{(2-q) H^{2}}{3 \Omega_{m 0} H_{0}^{2}}-\frac{1}{2}(1+z)^{3}\right], \\
& \left(\frac{d \phi}{d z}\right)^{2}=M_{p l}^{2}\left[\frac{2(1+q)}{(1+z)^{2}}-\frac{3 \Omega_{m 0} H_{0}^{2}}{H^{2}}(1+z)\right],
\end{aligned}
$$

where, $\Omega_{m 0} \equiv \frac{\rho_{m 0}}{3 M_{p l}^{2} H_{0}^{2}}$ is the dimensionless energy density, $H_{0}$ is the Hubble constant and the subscript 0 denotes the present value of a quantity at the redshift $\mathrm{z}=0$.

In order to realize the reconstruction $V(\phi)$ from the parameterized deceleration parameter $q(z)$, next we must deal with $H(z)$. The derivative of the Hubble parameter $H(z)$ with regard to the time $\mathrm{t}$ is $\dot{H}=-(1+q) H^{2}$. Then there exists a contact between $H(z)$ and $q(z)$ in virtue of an integration:

$$
\begin{aligned}
H(z) & =H_{0} \exp \int_{0}^{z}(1+q(u)) d \ln (1+u) \\
& =H_{0} f(z)
\end{aligned}
$$

where, $f(z) \equiv \exp \int_{0}^{z}(1+q(u)) d \ln (1+u)$. Hence, we 
tions for $V(z)$ and $\left(\frac{d \phi}{d z}\right)^{2}$ in term of $q(z)$ :

$$
\begin{aligned}
& V(\phi(z))=\rho_{m 0}\left[\frac{(2-q)}{3 \Omega_{m 0}} f^{2}(z)-\frac{1}{2}(1+z)^{3}\right] \\
& \left(\frac{d \phi}{d z}\right)^{2}=M_{p l}^{2}\left[\frac{2(1+q)}{(1+z)^{2}}-3 \Omega_{m 0}(1+z) f^{-2}(z)\right]
\end{aligned}
$$

We define the dimensionless quantities $\tilde{V} \equiv \frac{V}{\rho_{m 0}}$ and $\tilde{\phi} \equiv \frac{\phi}{M_{p l}}$. The reconstructed equations (10) and (11) can then be ulteriorly written as

$$
\begin{aligned}
& \tilde{V}(\tilde{\phi}(z))=\frac{(2-q)}{3 \Omega_{m 0}} f^{2}(z)-\frac{1}{2}(1+z)^{3}, \\
& \frac{d \tilde{\phi}}{d z}=-\sqrt{\frac{2(1+q)}{(1+z)^{2}}-3 \Omega_{m 0}(1+z) f^{-2}(z)} .
\end{aligned}
$$

These are the main results in our paper, which reveal the relation between the potential of the scalar field $V(\phi)$ and the deceleration parameter $q(z)$. If we provide an effective parameterized deceleration parameter $q(z)$, we can obtain the reconstruction of the potential $V(\phi)$ by the equations (12) and (13). Here, we have completed the extraction and chosen the expression with the minus, namely $\dot{\phi}<0$, in fact which has no effect to the result. If $\dot{\phi}>0$, we only need to change the definition of $\phi$ to $-\phi$.

In addition, we present the state parameter $w_{\phi}$, the dimensionless energy density $\Omega_{\phi}, \Omega_{m}$ on $q(z)$ :

$$
\begin{aligned}
& w_{\phi}=\frac{P_{\phi}}{\rho_{\phi}}=\frac{2 q-1}{3\left[1-\Omega_{m 0}(1+z)^{3} f^{-2}(z)\right]}, \\
& \Omega_{m}=\Omega_{m 0}(1+z)^{3} f^{-2}(z), \\
& \Omega_{\phi}=1-\Omega_{m} .
\end{aligned}
$$

The concrete expressions of these parameters can be readily attained after we provide an effective parameterized deceleration parameter $q(z)$. As concerning the parameters in parameterized deceleration parameter $q(z)$, we are able to constrict them through the latest observations[20],[21].

In this paper, we consider two kinds of parameterized parameters as follows:

Parametrization 1: $q(z)=a+\frac{b z}{(1+z)^{2}}$

Just in order to write conveniently formulae, we define $g(z) \equiv(1+z)^{-2(1+a+b)} \exp \left[\frac{2 b z}{1+z}\right] \equiv f^{-2}(z)$. So we have for Parametrization 1:

$$
\begin{aligned}
& \tilde{V}(z)=\frac{2-a-\frac{b z}{(1+z)^{2}}}{3 \Omega_{m 0}} g^{-1}(z)-\frac{1}{2}(1+z)^{3}, \\
& \frac{d \tilde{\phi}}{d z}=-\frac{\sqrt{2\left[1+a+\frac{b z}{(1+z)^{2}}\right]-3 \Omega_{m 0}(1+z)^{3} g(z)}}{1+z}(1,8) \\
& 2\left[a+\frac{b z}{(1+z)^{2}}\right]-1
\end{aligned}
$$

$$
\begin{aligned}
& \Omega_{m}=\Omega_{m 0}(1+z)^{3} g(z), \\
& \Omega_{\phi}=1-\Omega_{m} .
\end{aligned}
$$

Parametrization 2: $q(z)=\frac{1}{2}+\frac{a z+b}{(1+z)^{2}}$

It's the same to the case above and we define $h(z) \equiv \exp \left[-b-\frac{a z^{2}-b}{(1+z)^{2}}\right]$ for convenience, then obtaining

$$
\begin{aligned}
& \tilde{V}(z)=\left[\frac{\frac{3}{2}-\frac{a z+b}{(1+z)^{2}}}{3 \Omega_{m 0} h(z)}-\frac{1}{2}\right](1+z)^{3} \\
& \frac{d \tilde{\phi}}{d z}=-\frac{\sqrt{2\left[\frac{3}{2}+\frac{a z+b}{(1+z)^{2}}\right]-3 \Omega_{m 0} h(z)}}{1+z} \\
& w_{\phi}=\frac{2\left[\frac{1}{2}+\frac{a z+b}{(1+z)^{2}}\right]-1}{3\left[1-\Omega_{m 0} h(z)\right]} \\
& \Omega_{m}=\Omega_{m 0} h(z) \\
& \Omega_{\phi}=1-\Omega_{m}
\end{aligned}
$$

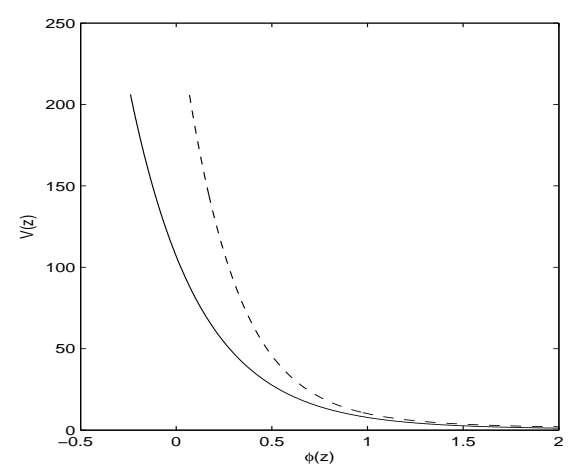

Fig.1 The evolution of the reconstructed potentials with the scalar field $\phi$.

We plot the potentials $V(\phi)$ in Fig. 1 and the evolution of the cosmological parameters in Fig. 2, 3. We get the values of the parameters by referring to [20] and taking $\Omega_{m 0}=0.3$ as a priority. 


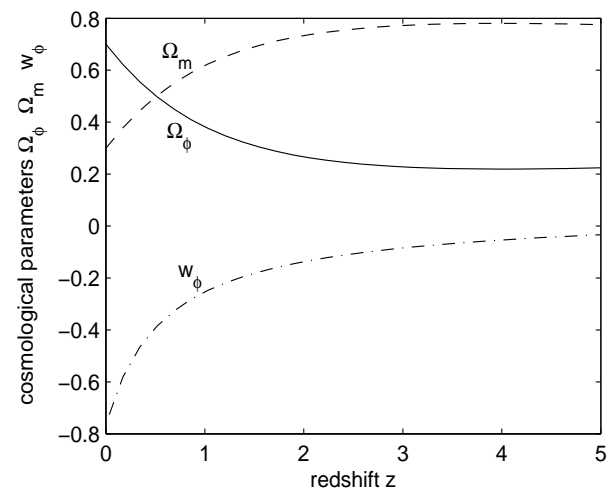

Fig. 2 The evolution of the cosmological parameters for Parametrization 1 with the redshift $z$, where, $a=-0.3$ and $b=0.8$ are used according to the observations constraint.

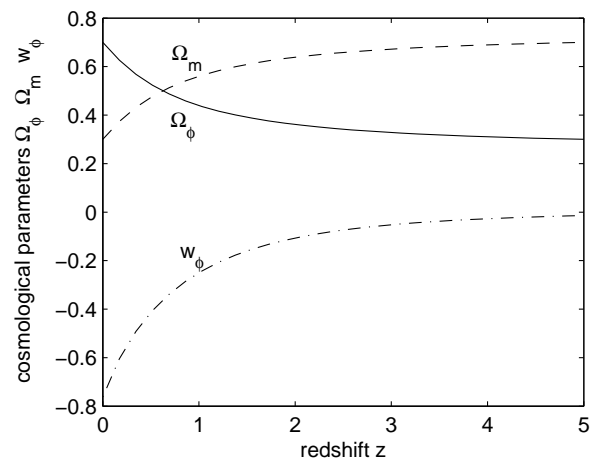

Fig.3 The evolution of the cosmological parameters for Parametrization 2 with the redshift $z$, where, $a=0.1$ and $b=-0.8$ are used according to the observations constraint.

In theory, we can get the function $\phi(z)$ by integrating the differential equation (13) for a concrete $q(z)$. Then the expression of $z$ with respect to $\phi$ can be obtained. After substituting $z(\phi)$ into the equation (12), we are able to get the analytical formula of the reconstructed potential. However, as we have seen above, the differential equations are too intricate to be integrated analytically in our considered cases. There- with the help of the initial condition $\phi_{0}=2$. In fact, the value of $\phi_{0}$ can't change the shape or trend of the potential $V(\phi)$. It just leads to shift the graphics horizontally. From the Fig. 1, it's demonstrated that the potentials decrease with the evolving scalar field $\phi$ and have the similar behaviors for the two kinds of examples at the late period. By our numerical evaluation, we discover that the evolving scalar field $\phi$ linearly runs away as the redshift $z$ increases. So the Figure 1 also presents the change rates of the reconstructed potential $V(\phi)$ are different between high redshift and low redshift, the former being faster than the latter.

Next, we plan to consider a simply form $q=$ constant, which is tenable within a small extension. Inserting it into the equations (12) and (13), we can hold on by considering discretely at low redshift and high one. For the low redshift, we can obtain an approximate analytic exponential expression:

$$
V(\phi)=\frac{2-q_{c}}{3 \Omega_{m 0}} \exp \left[\frac{-\left(2 q_{c}-1\right)\left(\phi-\phi_{0}\right)}{\sqrt{2\left(1+q_{c}\right)-3 \Omega_{m 0}}}\right]
$$

At high redshift, the result is different from that at low redshift. The formula is in square law:

$$
V(\phi)=\frac{\left(2-q_{c}\right)\left(q_{c}-\frac{1}{2}\right)^{2}}{4\left(1+q_{c}\right)^{4}}\left(\phi-\phi_{0}\right)^{2}
$$

It's found that this expression is independent on the value of the dimensionless matter density $\Omega_{m 0}$.

\section{Conclusions}

In summary, the dark energy potentials can be reconstructed from parameterized deceleration parameters. For the two examples given above, we obtain the evolution of the reconstructed potential $V(\phi)$, the dimensionless energy density $\Omega_{\phi}, \Omega_{m}$ and the state parameter $w_{\phi}$ for a given parameterized deceleration parameter $q(z)$. The result shows that the reconstructed potentials $V(\phi)$ decrease with the evolving scalar field $\phi$, namely that the reconstructed potentials $V(\phi)$ have the direction of going down with the expansion of the universe. Also it's found that the change rates of the reconstructed potential $V(\phi)$ are obviously diverse, which at $\phi<1$ is quicker than that at $\phi>1$. For the two cases, the variational trends of the reconstructed potentials $V(\phi)$ are very similar at the late period. In addition, analytically evaluating the equations (12) and (13) with $q=$ constant, we obtain two different approximate expressions and find the formula (28) don't contain the dimensionless 
concerned, we establish a relation between the deceleration parameter and the effective field theory in our means. With the progress of the observation tech- niques in the future, the parameters in $q(z)$ can be constrained more precisely, which can go far towards understanding the nature of dark energy for us.

\section{References}

[1] Riess A G, Filippenko A V, Challis P, Clocchiattia A, Diercks A, Garnavich P M, Gilliland R L, Hogan C J, Jha S, Kirshner R P, Leibundgut B, Phillips M M, Reiss D, Schmidt B P, Schommer R A, Smith R C, Spyromilio J, Stubbs C, Suntzeff N B and Tonry J 1998 Astrophy. J. 1161009 (astro-ph/9805201).

[2] Spergel D N, Verde L, Peiris H V, Komatsu E, Nolta M R, Bennett C L, Halpern M, Hinshaw G, Jarosik N, Kogut A, Limon M, Meyer S S, Page L, Tucker G S, Weiland J L, Wollack E and Wright E L 2003 Astrophys. J. Suppl. 148175

[3] Tegmark M, Strauss M A, Blanton M R, Abazajian K, Dodelson S, Sandvik H, Wang X M, Weinberg D H, Zehavi I, Bahcall N A, Hoyle F, Schlege D, Scoccimarro R, Vogeley M S, Berlind A, Budavari T, Connolly A, Eisenstein D J, Finkbeiner D, Frieman J A, Gunn J E, Hui L, Jain B, Johnston D, Kent S, Lin H, Nakajima R, Nichol R C, Ostriker J P, Pope A, Scranton R, Seljak U, Sheth R K, Stebbins A, Szalay A S, Szapudi I, Xu Y Z, Annis J, Brinkmann J, Burles S, Castander F J, Csabai I, Loveday J, Doi M, Fukugita M, Gillespie B, Hennessy G, Hogg D W, Ivezic Z, Knapp G R, Lamb D Q, Lee B C, Lupton R H, McKay T A, Kunszt P, Munn J A, O'Connell L, Peoples J, Pier J R, Richmond M, Rockosi C, Schneider D P, Stoughton C, Tucker D L, Vanden Berk D E, Yanny B and York D G 2004 Phys. Rev. D. 69103501.

[4] Starobinsky A A 1980 Phys. Lett. B. 9199.

Nojiri S and Odintsov S D 2003 Phys. Rev. D. 68123512.

Carroll S M, Duvvuri V, Trodden M and Turner M S 2004 Phys. Rev. D. 70043528.

Capozziello S, Cardone V F and Salzano V 2008 Phys. Rev. D. 78063504 .

Nojiri S and Odintsov S D 2008 Phys. Rev. D. 77026007.

[5] Capozziello S, Cardone V F and Troisi A 2005 Phys. Rev. D. 71043503 .

Wu X and Zhu Z H 2007 Phys. Lett. B. 660293.

[6] Ratra B and Peebles P J E 1988 Phys. Rev. D. 373406. Turner M S and White M 1997 Phys. Rev. D. 564439.

Caldwell R R, Dave R and Steinhardt P J 1998 Phys. Rev. Lett. 801582.

Steinhardt P J, Wang M L and Zlatev I 1999 Phys. Rev. D. 59123504

Sahni V and Starobinsky A A 2000 Int. J. Mod. Phys. D 9373.

Zhai X H and Zhao Y B 2006 Chin. Phys. 152465.

Zhao W 2007 Chin. Phys. 162894.
[7] Caldwell R R 2002 Phys. Lett. B. 54523.

Weinberg N N 2003 Phys. Rev. Lett. 91071301.

Nojiri S and Odintsov S D 2005 Phys. Rev. D. 72023003.

[8] Feng B, Wang X L and Zhang X M 2005 Phys. Lett. B. 60735 .

Guo Z K, Piao Y S, Zhang X M and Zhang Y Z 2005 Phys. Lett. B. 608177.

Setare M R 2006 Phys. Lett. B. 641130.

Setarea M R and Saridakis E N 2008 Phys. Lett. B. 668 177

[9] Kamenshchik A Y, Moschella U and Pasquier V 2001 Phys. Lett. B. 511265.

Bento M C, Bertolami O and Sen A A 2002 Phys. Rev. D. 66043507.

Bento M C, Bertolami O, Reboucas M J and Silva P T 2006 Phys. Rev. D. 73043504.

[10] Li M 2004 Phys. Lett. B. 6031.

Gong Y G 2004 Phys. Rev. D. 70064029.

Wang B, Abdalla E and Su R K 2005 Phys. Lett. B. 611 21.

[11] Randall L and Sundrum R 1999 Phys. Rev. Lett. 834690.

Zhu Z H and Alcaniz J S 2005 Astrophys. J 620711.

Pires N and Zhu Z H 2006 Phys. Rev. D. 73123530.

Tao B X, Ji S Y and Li F Q 2004 Chin. Phys. 131830.

[12] Sahni V and Starobinsky A A 2006 Int. J. Mod. Phys. D 152105 (astro-ph/0610026).

[13] Huterer D and Turner M S 2001 Phys. Rev. D. 64123527. Gong Y G and Zhang Y Z 2005 Phys. Rev. D. 72043518.

Gong Y G and Wang A Z 2007 Phys. Rev. D. 75043520.

[14] Wang Y and Freese K 2006 Phys. Lett. B. 632449.

[15] Gong Y G and Wang A Z 2006 Phys. Rev. D. 73083506.

[16] Sahlen M, Liddle A R and Parkinson D 2005 Phys. Rev. D. 72083511.

Sahlen M, Liddle A R and Parkinson D 2007 Phys. Rev. D. 75023502 .

[17] Guo Z K, Ohta N and Zhang Y Z 2005 Phys. Rev. D. 72 023504 (astro-ph/0505253).

[18] Guo Z K, Ohta N and Zhang Y Z 2007 Mod. Phys. Lett. A 22883 (astro-ph/0603109).

[19] Zhang C W, Xu L X, Chang B R and Liu H Y astro-ph/0703515

[20] Xu L X, Zhang C W, Chang B R and Liu H Y astro-ph/0701519

[21] Lu J B, Xu L X, Liu M L and Gui Y X 2009 Chin. Phys. $B 181711$. 